Article

\title{
Anomalous Sun Flyby of 1I/2017 U1 ('Oumuamua)
}

\author{
Klaus Wilhelm ${ }^{1, \dagger}$ and Bhola N. Dwivedi ${ }^{2, *, t, \ddagger}$ \\ 1 Max-Planck-Institut für Sonnensystemforschung (MPS), Justus-von-Liebig-Weg 3, \\ 37077 Göttingen, Germany; wilhelm@mps.mpg.de \\ 2 Department of Physics, Indian Institute of Technology, Banaras Hindu University, Varanasi 221005, India \\ * Correspondence: bnd.app@iitbhu.ac.in \\ + These authors contributed equally to this work. \\ $\ddagger$ Retired Professor.
}

Received: 12 October 2020; Accepted: 3 December 2020; Published: 7 December 2020

\begin{abstract}
The findings of Micheli et al. (Nature 2018, 559, 223-226) that 1I/2017 U1 ('Oumuamua) showed anomalous orbital accelerations have motivated us to apply an impact model of gravity in search for an explanation. A small deviation from the $1 / r$ potential, where $r$ is the heliocentric distance, is expected for the gravitational interaction of extended bodies as a consequence of this model. This modification of the potential results from an offset of the effective gravitational centre from the geometric centre of a spherically symmetric body. Applied to anomalous Earth flybys, the model accounts for energy gains relative to an exact Kepler orbit and an increased speed of several spacecraft. In addition, the flat rotation profiles of eight disk galaxies could be explained, as well as the anomalous perihelion advances of the inner planets and the asteroid Icarus. The solution in the case of 'Oumuamua is also based on the proposal that the offset leads to an approach and flyby trajectory different from a Kepler orbit without postulating cometary activity. As a consequence, an adjustment of the potential and centrifugal orbital energies can be envisaged outside the narrow uncertainty ranges of the published post-perihelion data without a need to re-analyse the original data. The observed anomalous acceleration has been modelled with respect to the orbit solutions JPL 16 and "Pseudo-MPEC" for 1I/‘Oumuamua.
\end{abstract}

Keywords: gravitational fields; asteroids; anomalous sun flyby; Solar System

PACS: 96.12.Fe; 96.30.Ys; 96.90.+c

\section{Introduction}

The astronomical body designated as 1I/2017 U1 ('Oumuamua) is the first object that has been observed during its passage through the Solar System from the interstellar environment. It was detected by Robert Weryk on 19 October 2017 with the Pan-STARRS telescope system in Hawaii [1]. Observations on 30 October 2017 of the lightcurve and its variation indicated a rotation period of 'Oumuamua of more than five hours [2]. Belton et al. reported in ref. [3] rotation periods near four and nine hours combined with an excited spin state of 'Oumuamua, cf. also ref. [4-7]. The findings by Micheli et al. [8] that 'Oumuamua's path deviates from a Kepler orbit resulted in a number of publications with different proposals to account for the additional acceleration. Cometary activity and the recoil resulting from outgassing would be a natural explanation, and is, indeed, "the most plausible physical model" [8]. However, even with long equivalent exposure times no cometary coma or tail activity could be detected [2,9]. The inactivity was also confirmed by Spitzer observations on 21 November 2017 [10], and Katz expressed in ref. [11] “... skepticism of the reported non-gravitational acceleration." Thus important problems remain, because many other observations showed neither 
cometary activity nor was any meteor activity detected on Earth, cf. [8,12-15]; in addition, the shape, consistence and origin of 'Oumuamua are still debated, cf. [16-32].

Solar radiation pressure could produce an excess radial acceleration with a $1 / r^{2}$ dependence, where $r$ is the heliocentric distance, if the object has a large surface and a very low mass according to refs. $[23,33]$. The authors of the latter reference even suggest as a possibility that a lightsail could be of “artificial origin". However, McNeil et al. [34] estimated a density of approximately $2000 \mathrm{~kg} \mathrm{~m}^{-3}$ for 'Oumuamua, a typical value for asteroids, cf. [35]. A density of this amount is hardly consistent with a lightsail. Radio SETI observations detected no signal [36].

The observation of an interstellar object in the Solar System raises the question with regard to where it might have come from. Potential sources have been indicated by [37-39], although the authors of ref. [37] point out that non-gravitational accelerations on the outbound path pose severe difficulties in determining the approach geometry.

In view of the many open questions concerning the nature of 'Oumuamua, we discuss in the following sections, whether a modification of the $1 / r$ gravitational potential and possible consequences could provide an answer to the anomalous Sun flyby of 1I/2017 U1 ('Oumuamua). Deviations from expected trajectories of artificial spacecraft had previously been deduced from Earth flyby observations, which were also not fully consistent with Newton's theory of gravity based on a potential exactly proportional to the inverse distance [40-44]. Many studies have been performed to solve this problem, e.g., in refs. [45-49].

We suggested that the interaction between gravitating bodies is affected by mass conglomerations according to an impact model of gravity [50,51] — based on the ideas of Nicolas Fatio de Duillier [52-54]. It results in a modification of the $1 / r$ potential $U$ for extended bodies, such as the Sun and the Earth. This could qualitatively explain the anomalous energy gain during Earth flybys [55]. Another application of the graviton impact model in the context of large masses could demonstrate a physical process to explain the anomalous rotation curves of disk galaxies [56,57]. The model has also successfully been applied to explain the secular perihelion advances of the inner planets and the asteroid Icarus with the result that an offset of $\rho_{\odot}=(4400 \pm 500) \mathrm{m}$ in the instantaneous direction to an orbiting body is required to explain the observed perihelion advances [58].

In this paper, we will consider our impact model for the gravitational interaction of 1I/2017 U1 ('Oumuamua) with the Sun. Since the Sun is not a gravitational point source, but has a nominal radius of $1 \mathcal{R}_{\odot}^{N}=6.957 \times 10^{8} \mathrm{~m}$, cf. [59]. The effective gravitational centre is, therefore, not expected to coincide with the geometric centre-even if a spherical symmetry of the Sun is assumed-but is situated on a sphere with radius $\rho_{\odot}$ around the centre, cf. [51]. We had thought that the observed anomalous acceleration of 'Oumuamua might also be a consequence of this offset. The calculations in the following sections ${ }^{1}$ show, however, that the effect of the energy gain with reasonable values of $\rho_{\odot}$ is too small to cause directly the unexpected acceleration, because of the large radial distances involved.

An indirect approach can, however, be pursued to adjust the orbit calculations. It still depends on the anomalous energy gain near perihelion, because it allows us to question the narrow ranges of the published observational uncertainties presented in Table 1 and to define adjusted trajectories outside these ranges which yield the required accelerations with respect to the expected motion of 'Oumuamua. It is to be noted here that we do not attempt to analyse original data, instead we use the reported orbit characteristics.

1 Most of the formulae needed are taken from Landau and Lifshitz in ref. [60]. Their original equation numbers are added in square brackets. The equations have only been modified to comply with the specific nomenclature used here. For instance, the effective potential energy Equation (3) originally reads

$$
U_{\mathrm{eff}}=-\frac{\alpha}{r}+\frac{M^{2}}{2 m r^{2}} .
$$




\section{Results}

\subsection{Modification of the Gravitational Potential}

The reduced mass $m$ of the two-body system Sun-'Oumuamua can be approximated by the mass $m_{\mathrm{Ou}}$ of 'Oumuamua according to

$$
\text { [13.4] } m=\frac{M_{\odot} m_{\mathrm{Ou}}}{M_{\odot}+m_{\mathrm{Ou}}} \approx m_{\mathrm{Ou}} .
$$

All calculations are, however, performed for a normalized reduced mass of $m=1 \mathrm{~kg}$ leading to specific quantities, such as, the specific angular momentum $\mathcal{M}$ and the specific approach energy $\mathcal{E}_{\infty}$ in the next sections. The use of these specific quantities eliminates the unknown mass of the attracted body and simplifies all equations.

The gravitational potential of the system can then be written as a specific quantity and would read under the assumption of the Sun as a point source:

$$
[15.1] \quad \mathcal{U}(r)=-\frac{\varepsilon}{r}
$$

where $\varepsilon=1.3271244 \times 10^{20} \mathrm{~m}^{3} \mathrm{~s}^{-2}$ is the nominal solar mass parameter [59] and $r=|\boldsymbol{r}|$ is the length of the heliocentric position vector. According to CODATA 2018, the gravitational constant is $G_{\mathrm{N}}=(6.67430 \pm 0.00015) \times 10^{-11} \mathrm{~m}^{3} \mathrm{~kg}^{-1} \mathrm{~s}^{-2}$ and gives a solar mass of $M_{\odot}=1.988409 \times 10^{30} \mathrm{~kg}$. The specific effective potential energy equation then becomes

$$
\mathcal{U}_{\mathrm{eff}}(r)=-\frac{\varepsilon}{r}+\frac{\mathcal{M}^{2}}{2 r^{2}}
$$

where the last term is the specific centrifugal energy.

Equation (2) has to be modified for bodies with large mass values, e.g., for the Sun, according to our gravitational interaction model. If the distance of the orbiting body from the Sun is always much greater than $1 \mathcal{R}_{\odot}^{N}$, the displacement $\rho_{\odot}$ of the effective gravitational centre mentioned in Section 1 can be assumed to be parallel to the radius vector $r$ in the direction of the orbiting body and, consequently, the specific potential energy will be

$$
\mathcal{U}_{\mathrm{mod}}=\frac{-\varepsilon}{r-\rho_{\odot}}=\frac{-\varepsilon\left(r+\rho_{\odot}\right)}{\left(r-\rho_{\odot}\right)\left(r+\rho_{\odot}\right)}=\frac{-\varepsilon\left(r+\rho_{\odot}\right)}{r^{2}-\rho_{\odot}^{2}}
$$

and can be approximated with $\rho_{\odot} \ll r$ by

$$
\mathcal{U}_{\text {mod }} \approx-\frac{\varepsilon}{r}-\frac{\varepsilon \rho_{\odot}}{r^{2}}
$$

The physical process causing the offset $\rho_{\odot}$, described in refs. [50,51], is that multiple interactions of the gravitons with the massive body occur before they can escape .

\subsection{Observations of 'Oumuamua}

The published orbit characteristics of 'Oumuamua considered in this study are compiled as data arcs No. 1 to 4 in Tables 1 and 2 from the corresponding references. This selection of different solutions out of many more evaluations available in the literature is listed to show the history of the observations and the resulting orbital parameters.

We suggest that a modification of the gravitational potential $\mathcal{U}(r)$ in Equation (2) to $\mathcal{U}_{\text {mod }}$ is required as given in Equation (4) for an adequate evaluation of the data arcs. A first attempt is made in Section 2.3 by varying the offset $\rho_{\odot}$ without changing $\mathcal{E}_{\infty}$ of orbit No. 4 in Table 2 . Although it will turn out that the effect with reasonable offset values is very small for 'Oumuamua, the deviation from 
an exact $1 / r$ potential justifies to scrutinize in Section 2.4 the tight uncertainties given for the orbit parameters in Table 1. These uncertainties determined from data arcs starting more than a month after the perihelion passage might not be representative for the modified potential. An indication of this expectation is that the orbit determinations No. 1 to 4 each exceed the uncertainty limits of $e$ and $q$ of the previous solution. For No. 3, the following statement ${ }^{2}$, related to the anomalous trajectory, supports this prospect: "The behavior of these accelerations outside the observed data arc from 14 October 2017 to 2 January 2018 can only be assumed. Predictions outside this time interval, particularly prior to October 2017, could be much more uncertain than reported here." Bailer-Jones et al. in [37] also point out that it is challenging to estimate the inbound leg of the trajectory under these conditions.

From the published eccentricity values $e$ and perihelion distances $q$ of the orbits in Table 1 (rounded to significant digits), the other quantities have been calculated under the assumption of hyperbolic Kepler motions:

The "semi-axis" $a$ of the hyperbola, the semi-latus rectum $p$ and the specific approach energy $\mathcal{E}_{\infty}$ with

$$
\text { [15.9] } \quad a=\frac{q}{e-1}(\mathrm{a}) ; \quad p=q(e+1)(\mathrm{b}) ; \quad \mathcal{E}_{\infty}=\frac{\varepsilon}{2 a}(\mathrm{c}),
$$

the specific angular momentum and the eccentricity with

$$
\text { [15.4] } \mathcal{M}=\sqrt{\varepsilon p} \quad \text { (a) } \quad e=\sqrt{1+\frac{2 \mathcal{M}^{2} \mathcal{E}_{\infty}}{\varepsilon^{2}}}
$$

the approach speed at infinity with

$$
V_{\infty}=\sqrt{2 \mathcal{E}_{\infty}} \quad \text { cf. }[18.3(1)]
$$

and the impact parameter with

$$
h=\frac{\mathcal{M}}{V_{\infty}} \quad \text { cf. }[18.3(2)]
$$


Table 1. Orbit characteristics during the ‘Oumuamua Sun flyby for four data arc evaluations ${ }^{\text {a }}$, modified orbit No. 4 and adjusted orbits No. 3 and 4.

\begin{tabular}{|c|c|c|c|c|c|}
\hline \multicolumn{2}{|l|}{ No. of Data Arcs } & \multicolumn{4}{|c|}{ Orbital Parameters ${ }^{b}$} \\
\hline & Observation Times & $e$ & q/au & $q /\left(10^{10} \mathrm{~m}\right)$ & $a /\left(10^{11} \mathrm{~m}\right)$ \\
\hline 1 & 18 October 2017-24 October 2017 & $1.182 \pm 0.017$ & $0.2483 \pm 0.0068$ & 3.715 & 2.05 \\
\hline 2 & 14 October 2017-17 November 2017 & $1.1995 \pm 0.0002$ & $0.25534 \pm 0.00007$ & 3.81976 & 1.91476 \\
\hline 3 & 14 October 2017-02 January 2018 & $1.20113 \pm 0.00002$ & $0.255912 \pm 0.000007$ & 3.8283828 & 1.9034010 \\
\hline 4 & 14 October 2017-02 January 2018 & $1.20117 \pm 0.00001$ & $0.255923 \pm 0.000003$ & 3.8285550 & 1.9031583 \\
\hline Modified orbit $^{c}$ & $\rho \stackrel{\mathrm{Ou}}{\odot} / \mathrm{m}$ & $e_{\mathrm{Ou}}$ & & $q /\left(10^{10} \mathrm{~m}\right)$ & $a /\left(10^{11} \mathrm{~m}\right)$ \\
\hline 4 & 4900 & 1.20117 & & 3.8285550 & 1.9031583 \\
\hline 4 & $1 \times 10^{8}$ & 1.2016070 & & 3.8285550 & 1.9031583 \\
\hline Adjusted orbits ${ }^{d}$ & $\rho_{\odot} / \mathrm{m}$ & $e_{\text {adj }}$ & & $q_{\mathrm{adj}} /\left(10^{10} \mathrm{~m}\right)$ & $a_{\mathrm{adj}} /\left(10^{11} \mathrm{~m}\right)$ \\
\hline 3 & 4900 & 1.2017066 & & 3.8360937 & 1.9018190 \\
\hline 4 & 4900 & 1.2018090 & & 3.8357945 & 1.9007052 \\
\hline
\end{tabular}

a Data (shortened to significant values) selected from the following references: No. 1: JPL Small-Body Database; JPL 1; producer Otto Matic; solution date 24 October 2017 (last accessed: 20 February 2019). No. 2: JPL 14; producer Davide Farnocchia; solution date 21 November 2017, cf. https://web.archive.org/web/20171122233227/ https://ssd.jpl.nasa.gov/ sbdb.cgi?sstr=2017U1; cad=1 (last accessed: 25 October 2020). No. 3: JPL 16; producer Davide Farnocchia; solution date 26 June 2018; cf. https:/ / ssd.jpl.nasa. gov/sbdb.cgi?sstr=1I (last accessed: 20 October 2020). No. 4: "Pseudo-MPEC" for 1I/‘Oumuamua; created 7 November 2018; IAU Minor Planet Center (MPC), cf. https://www.projectpluto.com/temp/ 2017u1.htm (last accessed: 10 November 2020). ${ }^{\mathrm{b}}$ Eccentricities: $e, e_{\mathrm{Ou}}$ and $e_{\mathrm{adj}}$; perihelion distances: $q$ and $q_{\mathrm{adj}} ; 1 \sigma$ uncertainties of $e$ and $q ;$;semi axes" $a$ and $a_{\text {adj }}$ calculated with Equations (6)a and (20)b; the astronomical unit $1 \mathrm{au}=149597870700 \mathrm{~m} \mathrm{[61].}{ }^{\mathrm{C}}$ Assuming a realistic offset $\rho_{\odot}$ and an unrealistic large one. Since $\mathcal{E}_{\infty}$ and the flyby distance are assumed to be constant, the "semi axis" $a$ and the perihelion $q$ have not changed either; violating Equation (6)a in the non-Keplerian orbits. ${ }^{\mathrm{d}}$ By adjusting $\mathcal{E}_{\text {adj }}$ and $\mathcal{M}_{\text {adj; }}$ see Equations (20)a-c. 
Table 2. Additional orbit characteristics derived from the eccentricities and perihelion distances of the data arcs No. 1 to 4 as well as for modified orbit No. 4 and adjusted orbits No. 3 and 4 in Table 1.

\begin{tabular}{|c|c|c|c|c|c|c|}
\hline & $\begin{array}{l}\text { Specific Approach } \\
\text { Energy }\end{array}$ & $\begin{array}{l}\text { Specific Angular } \\
\text { Momentum }\end{array}$ & $\begin{array}{l}\text { Semi-Latus } \\
\text { Rectum }\end{array}$ & $\begin{array}{l}\text { Speed } \\
\text { at Infinity }\end{array}$ & $\begin{array}{l}\text { Impact } \\
\text { Parameter }\end{array}$ & $\begin{array}{l}\text { Maximum } \\
\text { Speed }\end{array}$ \\
\hline No. of Data Arcs & $\mathcal{E}_{\infty} /\left(10^{8} \mathrm{~m}^{2} \mathrm{~s}^{-2}\right)$ & $\mathcal{M} /\left(10^{15} \mathrm{~m}^{2} \mathrm{~s}^{-1}\right)$ & $p /\left(10^{10} \mathrm{~m}\right)$ & $V_{\infty} /\left(\mathrm{m} \mathrm{s}^{-1}\right)$ & $h /\left(10^{11} \mathrm{~m}\right)$ & $V_{\text {peri }} /\left(\mathrm{m} \mathrm{s}^{-1}\right)$ \\
\hline 1 & 3.242 & 3.279 & 8.104 & 25465 & 1.288 & 88281 \\
\hline 2 & 3.46551 & 3.33914 & 8.40153 & 26327 & 1.26834 & 87418 \\
\hline 3 & 3.4861871 & 3.3441550 & 8.4267827 & 26405.254 & 1.2664733 & 87351.636 \\
\hline 4 & 3.4866318 & 3.3442565 & 8.4272947 & 26406.938 & 1.2665118 & 87350.359 \\
\hline \multirow[t]{2}{*}{ See } & Equation (6)c & Equation (7)a & Equation (6)b & Equation (8) & Equation (9) & Equation (12)c \\
\hline & Maximum & $1 \sigma$ values $^{\text {a }}$ & & & & \\
\hline 3 & 3.4866430 & 3.3442145 & & & & \\
\hline \multirow[t]{2}{*}{4} & 3.4868587 & 3.3442853 & & & & \\
\hline & & Modified & quantities ${ }^{b}$ & & & \\
\hline \multirow[t]{2}{*}{4} & 3.4866318 & 3.3482329 & 8.4289735 & 26406.938 & 1.2679369 & 87454.221 \\
\hline & & Adjusted & quantities $^{\mathrm{c}}$ & & & \\
\hline 3 & 3.4890871 & 3.3479566 & 8.4459528 & 26416.234 & 1.2673860 & 87275.151 \\
\hline 4 & 3.4911318 & 3.3479039 & 8.4456870 & 26423.973 & 1.2669949 & 87280.586 \\
\hline
\end{tabular}

${ }^{a}$ Calculated with the help of the uncertainty values in Table 1. ${ }^{\mathrm{b}}$ See Equations (10)-(14) . ${ }^{\mathrm{c}}$ See Equations (15)-(19). Relative increases of $\mathcal{E}_{\infty}^{\mathrm{max}}$ of $0.070 \%$ and $0.123 \%$ for orbits No. 3 and 4 , respectively, are required to model the anomalous accelerations and $0.111 \%$ and $0.108 \%$ for $\mathcal{M}^{\max }$. 


\subsection{Orbit Modification by Offset $\rho \odot^{\text {Ou }}$}

The laws of conservation of energy and angular momentum determine the motion of a body in a central gravitational configuration:

$$
[14.4] \quad \frac{1}{2}\left(\frac{\mathrm{d} r}{\mathrm{~d} t}\right)^{2}=\mathcal{E}_{\infty}-\mathcal{U}(r)-\frac{\mathcal{M}^{2}}{2 r^{2}}=\frac{1}{2} \dot{r}^{2}=\mathcal{E}_{\infty}+\frac{\varepsilon}{r}-\frac{\mathcal{M}^{2}}{2 r^{2}}
$$

where $\mathrm{d} r / \mathrm{d} t=\dot{r}$ is the radial velocity. This equation describes a Kepler orbit with an unmodified Equation (2). There is no radial velocity $\dot{r}_{\text {peri }}$ at perihelion, but the highest tangential speed $V_{\text {peri }}$. For a perihelion distance $q$ it is:

$$
\begin{array}{r}
\frac{1}{2} \dot{r}_{\text {peri }}^{2}=\mathcal{E}_{\infty}+\frac{\varepsilon}{q}-\frac{\mathcal{M}^{2}}{2 q^{2}}=0 \quad(\mathrm{a}) \\
\frac{1}{2} V_{\text {peri }}^{2}=\mathcal{E}_{\infty}+\frac{\varepsilon}{q}=\frac{\mathcal{M}^{2}}{2 q^{2}} \quad(\mathrm{~b}) \\
V_{\text {peri }}=\frac{\mathcal{M}}{q} \quad(\mathrm{c}) .
\end{array}
$$

With the modified potential $\mathcal{U}_{\text {mod }}$ of Equation (4) the Equation (10) reads:

$$
\frac{1}{2} \dot{r}_{\text {mod }}^{2}=\mathcal{E}_{\infty}+\frac{\varepsilon}{r-\rho_{\odot}^{\mathrm{Ou}}}-\frac{\mathcal{M}_{\mathrm{mod}}^{2}}{2 r^{2}}
$$

It should be noted that the denominator of the potential energy term depends on $\rho \odot{ }_{\odot}^{\mathrm{Ou}}$ for the modified orbit, whereas the one of the centrifugal energy term does not, because the angular momentum is defined about the centre of the solar mass.

A comparison with the Kepler orbit in Equation (10) can best be made at perihelion $q$, where $\dot{r}_{\text {peri }}=0$. From

$$
\mathcal{E}_{\infty}+\frac{\varepsilon}{q}-\frac{\mathcal{M}^{2}}{2 q^{2}}=\mathcal{E}_{\infty}+\frac{\varepsilon}{q-\rho_{\odot}^{\mathrm{Ou}}}-\frac{\mathcal{M}_{\mathrm{mod}}^{2}}{2 q^{2}}=0
$$

then follows an exact solution for $\mathcal{M}_{\text {mod }}$ using a constant $\mathcal{E}_{\infty}$ :

$$
\mathcal{M}_{\text {mod }}=q \sqrt{2\left(\mathcal{E}_{\infty}+\frac{\varepsilon}{q-\rho \mathrm{Ou}}\right)},
$$

which is a constant of the motion. Since at the start of the data arcs at a radial distance $r_{1} \gg \rho \odot$ Ou (cf. Equation (17) and Table 3) and beyond the modified orbit can be approximated by a Kepler orbit, we use Equation (7)b with $\mathcal{M}_{\text {mod }}$ to estimate the modified eccentricity $e_{\mathrm{OU}}$ for the orbit calculations in Section 2.4. The quality of the approximation can be checked after an estimate of $\rho$ Ou has been obtained (as listed in Tables 1 and 3). The initial conditions at $r_{1}$ can be defined by requiring $r_{1}=r_{1}^{\mathrm{Ou}}$ and $\dot{r}_{1} \leq \dot{r}_{1}^{\mathrm{Ou}}$, where the radial velocity can only be approximated, because a constant $\mathcal{E}_{\infty}$ leads to a constant $\dot{r}_{\text {mod }}^{\infty}$ with Equation (12). This equation will be evaluated 
in Section $2.4^{3}$ using the specific angular momentum $\mathcal{M}_{\text {mod }}$ obtained in Equation (14) with varying assumptions for $\rho \odot$ Ou until a reasonable anomalous acceleration fit has been achieved in Figure 1.

As can be seen from Table 3, an offset of $4900 \mathrm{~m}$ had only a minor effect on the anomalous accelerations and thus the effect is not sufficient to explain directly the anomalous acceleration found in ref. [8] for 'Oumuamua: "... which corresponds to a formal detection of non-gravitational acceleration with a significance of about $30 \sigma$." This detection allows a range of accelerations between $A_{1} /(r / \mathrm{au})^{2}$ and $A_{1} /(\mathrm{r} / \mathrm{au})$, "where $A_{1}$ is a free fit parameter" with a value of " $A_{1}=(4.92 \pm 0.16) \times 10^{-6} \mathrm{~m} \mathrm{~s}^{-2 "}$.

Since we see no reason as to why the solar flyby of 'Oumuamua the offset valid for the perihelion advances of the inner planets should be outside the range $\rho_{\odot}=(4400 \pm 500) \mathrm{m}$, we will use its maximum in the next section. The specific potential energy gain at perihelion in Equation (14) would be $\varepsilon \rho_{\odot} / q^{2}=444 \mathrm{~m}^{2} \mathrm{~s}^{-2}$ and the specific centrifugal energy is also increased by that amount ${ }^{4}$.

\subsection{Orbital Parameter Adjustments}

As outlined in Section 2.2, the uncertainties in the orbital parameters might be much larger than given in Table 1. In particular, we suspect that the relation between the specific approach energy in Equation (6)c and the specific angular momentum in Equation (7)a is not adequately constrained and, therefore, adjusted quantities outside the narrow limits can later be used in Equation (17). In Table 2, the energy and angular momentum quantities are given in detail together with other supplementary data.

The maxima of $\mathcal{E}_{\infty}$ and $\mathcal{M}$ have been obtained from the extreme values of $e$ and $q$ of the orbit solutions No. 3 and 4. Taking the above considerations into account, we argue that we are justified to increase the specific approach and centrifugal energies of the adjusted orbits beyond the maximum $1 \sigma$ values in Table 2 and compare them with orbits No. 3 and 4 in an attempt to model the anomalous accelerations on the data arcs. The details of the adjustment can only be estimated by a trial and error method to be explained below, after the specific approach energy had been increased by a certain amount $\Delta \mathcal{E}$ to

$$
\mathcal{E}_{\text {adj }}=\mathcal{E}_{\infty}+\Delta \mathcal{E}
$$

3 The physical process leading to the unexpected acceleration can be demonstrated by a comparison of Equations (10) and (12) at the heliocentric distance $r_{1}$ (the start of a data arc), where both radial velocities are assumed to be equal, and at a greater distance $r_{2}=r_{1}+\rho_{\odot}$. This implies:

$$
\frac{1}{2}\left(\dot{r}_{\text {mod, } 1}^{2}-\dot{r}_{1}^{2}\right)=\frac{\varepsilon}{r_{1}}+\frac{\varepsilon}{r_{1}-\rho_{\odot}}-\frac{\mathcal{M}_{\text {mod }}^{2}-\mathcal{M}^{2}}{2 r_{1}^{2}}=0 \text { and thus } \mathcal{M}_{\text {mod }}^{2}-\mathcal{M}^{2}=\frac{2 \varepsilon \rho_{\odot}}{1-\rho_{\odot} / r_{1}} .
$$

To find the specific effective potential energy change in a Kepler orbit and a modified one, we compare

$$
\frac{1}{2}\left(\dot{r}_{2}^{2}-\dot{r}_{1}^{2}\right)=\frac{\varepsilon}{r_{2}}-\frac{\mathcal{M}^{2}}{2 r_{2}^{2}}-\frac{\varepsilon}{r_{1}}+\frac{\mathcal{M}^{2}}{2 r_{1}^{2}} \text { and } \frac{1}{2}\left(\dot{r}_{\text {mod, } 2}^{2}-\dot{r}_{\text {mod, } 1}^{2}\right)=\frac{\varepsilon}{r_{2}-\rho_{\odot}}-\frac{\mathcal{M}_{\text {mod }}^{2}}{2 r_{2}^{2}}-\frac{\varepsilon}{r_{1}}+\frac{\mathcal{M}_{\text {mod }}^{2}}{2 r_{1}^{2}} .
$$

A lengthy calculation then shows that the decrease in the specific effective potential energy in the modified orbit is smaller than in the Kepler orbit by $2 \varepsilon \rho_{\odot}^{2} /\left[r_{1}\left(r_{1}^{2}-\rho_{\odot}^{2}\right)\right]$.

4 The Parker Solar Probe reached its first perihelion with $q_{\mathrm{PSP}} \approx 2.48 \times 10^{10} \mathrm{~m}$ on 6 November 2018 at 03:28 UTC (NASA: Parker Solar Probe Reports). This was about $1.35 \times 10^{10} \mathrm{~m}$ closer than 'Oumuamua. The specific potential energy gain would have been $\approx 1054 \mathrm{~m}^{2} \mathrm{~s}^{-2}$ with $\rho_{\odot}=4900 \mathrm{~m}$ and $\approx 2.15 \times 10^{7} \mathrm{~m}^{2} \mathrm{~s}^{-2}$ with $\rho_{\odot}^{\mathrm{Ou}}=1 \times 10^{8} \mathrm{~m}$. 
Although the variation of the specific angular momentum in the adjusted orbit equation

$$
\begin{array}{r}
\frac{1}{2} \dot{r}_{\text {adj }}^{2}=\mathcal{E}_{\text {adj }}+\frac{\varepsilon}{r_{\text {adj }}-\rho_{\odot}}-\frac{(\mathcal{M}+\Delta \mathcal{M})^{2}}{2 r_{\text {adj }}^{2}} \approx \mathcal{E}_{\infty}+\Delta \mathcal{E}+\frac{\varepsilon}{r_{\text {adj }}}+\frac{\varepsilon \rho_{\odot}}{r_{\text {adj }}^{2}}-\frac{(\mathcal{M}+\Delta \mathcal{M})^{2}}{2 r_{\text {adj }}^{2}}= \\
\mathcal{E}_{\infty}+\frac{\varepsilon}{r_{\text {adj }}}-\frac{(\mathcal{M}+\Delta \mathcal{M})^{2}-2 \varepsilon \rho_{\odot}-2 r_{\text {adj }}^{2} \Delta \mathcal{E}}{2 r_{\text {adj }}^{2}}=\mathcal{E}_{\text {adj }}+\frac{\varepsilon}{r_{\text {adj }}}-\frac{(\mathcal{M}+\Delta \mathcal{M})^{2}-2 \varepsilon \rho \odot}{2 r_{\text {adj }}^{2}}= \\
\mathcal{E}_{\text {adj }}+\frac{\varepsilon}{r_{\text {adj }}}-\frac{\mathcal{M}_{\text {adj }}^{2}}{2 r_{\text {adj }}^{2}},
\end{array}
$$

is, in principle, not dependent on that of the specific energy, we feel that the observations reflected in the orbit parameters No. 3 and 4 might provide some constraints:

(1) Assuming that the heliocentric distance $r$ of 'Oumuamua and its radial velocity $\dot{r}$ could best be established at the beginning of the observations, we require that the initial conditions agree for orbit No. 4 and the corresponding adjusted trajectory. By equating Equations (10) and (17) at $r_{1}=r_{1}^{\text {adj }}$

$$
\mathcal{E}_{\infty}+\frac{\varepsilon}{r_{1}}-\frac{\mathcal{M}^{2}}{2 r_{1}^{2}}=\mathcal{E}_{\infty}+\frac{\varepsilon}{r_{1}}-\frac{(\mathcal{M}+\Delta \mathcal{M})^{2}-2 \varepsilon \rho_{\odot}-2 r_{1}^{2} \Delta \mathcal{E}}{2 r_{1}^{2}},
$$

the same radial velocity $\dot{r}_{1}=\dot{r}_{1}^{\text {adj }}$ is valid for both trajectories at this distance. It can be seen that

$$
(\mathcal{M}+\Delta \mathcal{M})^{2}=\mathcal{M}^{2}+2 \varepsilon \rho_{\odot}+2 r_{1}^{2} \Delta \mathcal{E}
$$

and that the adjusted specific angular momentum as function of the fit parameter $\Delta \mathcal{E}$ is

$$
\mathcal{M}_{\mathrm{adj}}=\sqrt{(\mathcal{M}+\Delta \mathcal{M})^{2}-2 \varepsilon \rho_{\odot}}=\sqrt{\mathcal{M}^{2}+2 r_{1}^{2} \Delta \mathcal{E}},
$$

cf. Equation (17). It is important to note that the perihelion distance has to change accordingly. As there are no observations available for $q$, this cannot be checked and lead to a potential conflict.

(2) For the adjustment of orbit solution No. 3, we refer to a statement by Micheli et al. in the appendix of [8]: METHODS, Section Non-gravitational models. We interpret it to mean that "the non-gravitational acceleration on "Oumuamua on October 25 at" $r=1.4$ au was $2.7 \times 10^{-6} \mathrm{~m} \mathrm{~s}^{-2}$. Assuming that the radial velocity was well defined there by solution No. 3, we use a heliocentric distance $r_{\diamond}=1.4 \mathrm{au}=2.0943702 \times 10^{11} \mathrm{~m}$ instead of $r_{1}$ in Equation (19).

With corresponding values for $\mathcal{E}_{\text {adj }}$ and $\mathcal{M}_{\text {adj }}$, the adjusted (or modified) orbit characteristics in Table 1 would be defined by

$$
e_{\mathrm{adj}} \approx \sqrt{1+\frac{2 \mathcal{E}_{\mathrm{adj}} \mathcal{M}_{\mathrm{adj}}^{2}}{\varepsilon^{2}}}(\mathrm{a}) ; \quad a_{\mathrm{adj}}=\frac{\varepsilon}{2 \mathcal{E}_{\mathrm{adj}}}(\mathrm{b}) ; \quad q_{\mathrm{adj}} \approx a_{\mathrm{adj}}\left(e_{\mathrm{adj}}-1\right) \quad(\mathrm{c}),
$$

cf. Equations (7)b and (6)a,c. In Section 3 the quality of the approximations will be considered. 
In the next step, we have to establish the time dependence of the hyperbolical motions on the trajectories with parametric equations for the time $t$, the heliocentric distance $r$ and the heliocentric $x$ and $y$ coordinates given in ref. [60] $(\$ 15, \text { p. } 38)^{5}$ :

$$
\begin{aligned}
& t=\sqrt{\frac{a^{3}}{\varepsilon}}(e \sinh \chi-\chi)(\mathrm{a}) ; \quad r=a(e \cosh \chi-1)(\mathrm{b}) ; \\
& x=a(e-\cosh \chi) \quad(\mathrm{c}) ; \quad y=a \sqrt{e^{2}-1} \sinh \chi(\mathrm{d}),
\end{aligned}
$$

where the parameter $\chi$ varies from $-\infty$ to $+\infty$ for a complete flyby. The equations have to be applied for orbit characteristics $e$ and $a$ in Table 1, as well as for $e_{\text {adj }}$ and $a_{\mathrm{adj}}$, cf. Equations (20)a,b, both for orbits No. 3 and 4 under consideration. The same procedure will be used to obtain an estimate of $e_{\mathrm{Ou}}$ as a function of $\rho_{\odot}$ or $\rho \odot$ Ou .

As listed in Table 3 , the time $t_{1}$-the start time of the observations in seconds after the perihelion passage-should correspond to $\chi\{0\}$ and $t_{2}$, the end of the observations to $\chi\{100\}$ for orbits No. 3 and 4 . The formalism allows us to calculate the radial velocities $\dot{r}$ in Equation (10) for both data arcs between $t_{1}$ and $t_{2}$ at times $t\{i\}$ and positions $r\{i\}$ for equidistant values of the parameters $\chi\{i\}$ (i=0 to 100 ). This procedure yields 101 data points and 100 intervals in Equations (22) and (23) (A test with more intervals did not substantially improve the calculations). The same calculations have to be done for the modified and adjusted configurations of Equations (17) and (12) before hundred variations each of the velocity components

$$
\Delta \dot{r}(\chi\{i\})=\dot{r}(\chi\{i+1\})-\dot{r}(\chi\{i\}) \text { and } \Delta \dot{r}_{\text {adj }}\left(\chi_{\text {adj }}\{i\}\right)=\dot{r}_{\text {adj }}\left(\chi_{\text {adj }}\{i+1\}\right)-\dot{r}_{\text {adj }}\left(\chi_{\text {adj }}\{i\}\right) \text {, }
$$

can be determined for $i=0$ to 99 . The corresponding time intervals are

$$
\Delta t(\chi\{i\})=t(\chi\{i+1\})-t(\chi\{i\}) \text { and } \Delta t_{\text {adj }}\left(\chi_{\text {adj }}\{i\}\right)=t_{\text {adj }}\left(\chi_{\text {adj }}\{i+1\}\right)-t_{\text {adj }}\left(\chi_{\text {adj }}\{i\}\right) .
$$

The expected accelerations along the trajectories of No. 3 and 4 then are

$$
A_{3,4}^{\exp }\{i\}=\Delta \dot{r}(\chi\{i\}) / \Delta t(\chi\{i\}) .
$$

The adjusted accelerations are

$$
A_{3,4}^{\text {adj }}\{i\}=\Delta \dot{r}_{\text {adj }}\left(\chi_{\text {adj }}\{i\}\right) / \Delta t_{\text {adj }}\left(\chi_{\text {adj }}\{i\}\right)
$$

and $A_{4}^{\mathrm{Ou}}$, respectively. All accelerations are negative, i.e., decelerations, on the outbound path. Subtraction of the expected greater decelerations from the adjusted values yields the positive accelerations

$$
\Delta A_{3,4}^{\operatorname{adj}}\{i\}=A_{3,4}^{\operatorname{adj}}\{i\}-A_{3,4}^{\exp }\{i\}
$$

for $i=0$ to 99. By design, it is $t_{2}^{\text {adj }}-t_{1}^{\text {adj }}=t_{2}-t_{1}$ for both trajectories and $r_{1}^{\text {adj }}=r_{1}$ for No. 4 . Even for No. $3 r_{1}^{\text {adj }} \approx r_{1}$ is a good approximation. In addition, we have, with a regression coefficient $\beta_{r}=1.000201$, the relation $r_{\text {adj }}\{i\}-r_{1}^{\text {adj }}=\beta_{r}\left(r\{i\}-r_{1}\right)$. Both the $\Delta A_{3,4}\{i\}$ and $\Delta A_{3,4}^{\text {adj }}\{i\}$ can, therefore, be plotted in Figure 1 over the heliocentric distance $r$ for a comparison with the $A_{1} /(r / \mathrm{au})$ and $A_{1} /(r / \mathrm{au})^{2}$ fits.

5 Starting from Equations [14.4], cf. Equation (10), and [14.5] of ref. [60], Landau and Lifshitz obtained the time $t$ by integrating $\mathrm{d} t$ with the substitution $r+a=a e \cosh \chi$. 
Table 3. Boundaries of the trajectory calculations and anomalous acceleration results.

\begin{tabular}{|c|c|c|}
\hline Start of Observation a & Heliocentric Distance $r_{\diamond} b$ & End of Observation ${ }^{c}$ \\
\hline \multicolumn{3}{|l|}{ Data $\operatorname{arc}$ No. $3^{\text {d,e }} \quad$ with $\rho_{\odot}=4.9 \mathrm{~km}$} \\
\hline$t_{1}=3018127.7 \mathrm{~s}$ & $t_{\diamond}=4071215.1 \mathrm{~s}$ & $t_{2}=9933475.7 \mathrm{~s}$ \\
\hline$\chi\{0\}=1.0082868$ & $\chi(23)=1.1575382$ & $\chi\{100\}=1.6572059$ \\
\hline$t_{1}^{\text {adj }}=3018523.2 \mathrm{~s}$ & $t_{\diamond}^{\text {adj }}=4071591.1 \mathrm{~s}$ & $t_{2}^{\text {adj }}=9933871.2 \mathrm{~s}$ \\
\hline$\chi_{\text {adj }}\{0\}=1.0081679$ & $\chi_{\text {adj }}\{23\}=1.1574799$ & $\chi_{\text {adj }}\{100\}=1.6573503$ \\
\hline$r_{1}=1.6468369 \times 10^{11} \mathrm{~m}$ & $r_{\diamond}=2.0933441 \times 10^{11} \mathrm{~m}$ & $r_{2}=4.3098053 \times 10^{11} \mathrm{~m}$ \\
\hline $\begin{aligned} r_{1}^{\mathrm{adj}} & =1.6468869 \times 10^{11} \mathrm{~m} \\
\dot{r}_{1} & =43.550029 \mathrm{~km} / \mathrm{s}\end{aligned}$ & $\begin{aligned} r_{\diamond}^{\mathrm{adj}} & =2.0933173 \times 10^{11} \mathrm{~m} \\
\dot{r}_{\diamond} & =41.351855 \mathrm{~km} / \mathrm{s}\end{aligned}$ & $\begin{aligned} r_{2}^{\text {adj }} & =4.3100180 \times 10^{11} \mathrm{~m} \\
\dot{r}_{2} & =35.396199 \mathrm{~km} / \mathrm{s}\end{aligned}$ \\
\hline$\dot{r}_{1}^{\text {adj }}=43.545919 \mathrm{~km} / \mathrm{s}$ & $\dot{r}_{\diamond}^{\text {adj }}=41.351965 \mathrm{~km} / \mathrm{s}$ & $\dot{r}_{2}^{\text {adj }}=35.402111 \mathrm{~km} / \mathrm{s}$ \\
\hline$\Delta A_{1}^{\text {adj }}=5.6040 \times 10^{-6} \mathrm{~m} \mathrm{~s}^{-2}$ & $\Delta A_{\diamond}^{\text {adj }}=2.7008 \times 10^{-6} \mathrm{~m} \mathrm{~s}^{-2}$ & $\Delta A_{2}^{\text {adj }}=0.3714 \times 10^{-6} \mathrm{~m} \mathrm{~s}^{-2}$ \\
\hline \multicolumn{3}{|l|}{ Data arc No. $4^{\text {e,f }} \quad$ with $\rho_{\odot}=4.9 \mathrm{~km}$} \\
\hline$t_{1}=3018115.6 \mathrm{~s}$ & $t_{\diamond}=4071197.8 \mathrm{~s}$ & $t_{2}=9933463.6 \mathrm{~s}$ \\
\hline$\chi\{0\}=1.0083298$ & $\chi(23)=1.1575890$ & $\chi\{100\}=1.6572838$ \\
\hline$t_{1}^{\text {adj }}=3018289.0 \mathrm{~s}$ & $t_{\diamond}^{\text {adj }}=4071320.9 \mathrm{~s}$ & $t_{2}^{\text {adj }}=9933636.9 \mathrm{~s}$ \\
\hline$\chi_{\text {adj }}\{0\}=1.0084153$ & $\chi_{\text {adj }}\{23\}=1.1577639$ & $\chi_{\text {adj }}\{100\}=1.6577568$ \\
\hline$r_{1}=1.6468462 \times 10^{11} \mathrm{~m}$ & $r_{\diamond}=2.0933591 \times 10^{11} \mathrm{~m}$ & $r_{2}=4.3098798 \times 10^{11} \mathrm{~m}$ \\
\hline $\begin{aligned} r_{1}^{\mathrm{adj}} & =1.6468462 \times 10^{11} \mathrm{~m} \\
\dot{r}_{1} & =43.550712 \mathrm{~km} / \mathrm{s}\end{aligned}$ & $\begin{aligned} r_{\diamond}^{\text {adj }} & =2.0933621 \times 10^{11} \mathrm{~m} \\
\dot{r}_{\diamond} & =41.352677 \mathrm{~km} / \mathrm{s}\end{aligned}$ & $\begin{aligned} r_{2}^{\mathrm{adj}} & =4.3103702 \times 10^{11} \mathrm{~m} \\
\dot{r}_{2} & =35.397282 \mathrm{~km} / \mathrm{s}\end{aligned}$ \\
\hline$\dot{r}_{1}^{\mathrm{adj}}=43.550712 \mathrm{~km} / \mathrm{s}$ & $\dot{r}_{\diamond}^{\text {adj }}=41.356811 \mathrm{~km} / \mathrm{s}$ & $\dot{r}_{2}^{\text {adj }}=35.407342 \mathrm{~km} / \mathrm{s}$ \\
\hline$\Delta A_{1}^{\text {adj }}=5.3775 \times 10^{-6} \mathrm{~m} \mathrm{~s}^{-2}$ & $\Delta A_{\diamond}^{\text {adj }}=2.6246 \times 10^{-6} \mathrm{~m} \mathrm{~s}^{-2}$ & $\Delta A_{2}^{\text {adj }}=0.4234 \times 10^{-6} \mathrm{~m} \mathrm{~s}^{-2}$ \\
\hline \multicolumn{3}{|l|}{ Data $\operatorname{arc~No.~} 4^{\mathrm{g}} \quad$ with $\rho \stackrel{\mathrm{Ou}}{\odot}=4.9 \mathrm{~km}$} \\
\hline$\Delta A_{1}^{\mathrm{Ou}}=2.328 \times 10^{-10} \mathrm{~m} \mathrm{~s}^{-2}$ & $\Delta A_{\diamond}^{\mathrm{Ou}}=0.000 \times 10^{-10} \mathrm{~ms}^{-2}$ & $\Delta A_{2}^{\mathrm{Ou}}=0.000 \times 10^{-10} \mathrm{~m} \mathrm{~s}^{-2}$ \\
\hline \multicolumn{3}{|l|}{ Data $\operatorname{arc~No.~} 4^{\text {e,h }} \quad$ with $\rho \odot{ }_{\odot}^{\mathrm{Ou}}=100000 \mathrm{~km}$} \\
\hline $\begin{aligned} t_{1}^{\mathrm{Ou}} & =3018893.4 \mathrm{~s} \\
r_{1} & =1.6468462 \times 10^{11} \mathrm{~m}\end{aligned}$ & $\begin{aligned} t_{\diamond}^{\mathrm{Ou}} & =4072010.7 \mathrm{~s} \\
r_{\diamond} & =2.0933591 \times 10^{11} \mathrm{~m}\end{aligned}$ & $\begin{aligned} t_{2}^{\mathrm{Ou}} & =9934241.4 \mathrm{~s} \\
r_{2} & =4.3098798 \times 10^{11} \mathrm{~m}\end{aligned}$ \\
\hline $\begin{aligned} r_{1}^{\mathrm{Ou}} & =1.6468462 \times 10^{11} \mathrm{~m} \\
\dot{r}_{1} & =43.550712 \mathrm{~km} / \mathrm{s}\end{aligned}$ & $\begin{aligned} r_{\diamond}^{\mathrm{Ou}} & =2.0932806 \times 10^{11} \mathrm{~m} \\
\dot{r}_{\diamond} & =41.352677 \mathrm{~km} / \mathrm{s}\end{aligned}$ & $\begin{aligned} r_{2}^{\mathrm{Ou}} & =4.3095964 \times 10^{11} \mathrm{~m} \\
\dot{r}_{2} & =35.397282 \mathrm{~km} / \mathrm{s}\end{aligned}$ \\
\hline$\dot{r}_{1}^{\mathrm{Ou}}=43.539445 \mathrm{~km} / \mathrm{s}$ & $\dot{r}_{\diamond}^{\mathrm{Ou}}=41.345909 \mathrm{~km} / \mathrm{s}$ & $\dot{r}_{2}^{\mathrm{Ou}}=35.395830 \mathrm{~km} / \mathrm{s}$ \\
\hline$\Delta A_{1}^{\mathrm{Ou}}=6.3539 \times 10^{-6} \mathrm{~m} \mathrm{~s}^{-2}$ & $\Delta A_{\diamond}^{\mathrm{Ou}}=2.8207 \times 10^{-6} \mathrm{~m} \mathrm{~s}^{-2}$ & $\Delta A_{2}^{\mathrm{Ou}}=0.2517 \times 10^{-6} \mathrm{~m} \mathrm{~s}^{-2}$ \\
\hline
\end{tabular}

${ }^{a}$ Julian date 2458040.93936 (14 October 2017; 10:32:40.7 UTC, start of observations) and perihelion times of both orbits (see Notes $\mathrm{d}$ and e) define times $t_{1}$ by adjusting $\chi(0)$ in Equation (21)a. ${ }^{\mathrm{b}}$ For orbit No. 3 , we assumed $\dot{r}_{\diamond}^{\text {adj }} \approx \dot{r}^{\diamond}$ at $r_{\diamond}^{\text {adj }} \approx r_{\diamond}$ according to Constraint (2). The approximations could have been improved by smaller steps of the parameter $\chi$. ${ }^{c}$ JD 2458120.97811 (2 January 2018; 11:28:28.7 UTC, end of observations) defines times $t_{2}$ by adjusting $\chi(100)$. ${ }^{\mathrm{d}}$ Perihelion on 9 September 2017 12:10:32.5 UTC (JD 2458006.007321) defines time $t_{0}=0 \mathrm{~s}$ for No. 3 (JPL 16 for 1I/‘Oumuamua). Adjustment of $\chi_{\text {adj }}\{0\}$ by applying Constraint (2) above. ${ }^{\mathrm{e}}$ By an appropriate selection of the parameter limit $\chi_{\text {adj }}\{100\}$, the durations of all data arcs become $t_{2}-t_{1}=t_{2}^{\text {adj }}-t_{1}^{\text {adj }}=t_{2}^{\text {Ou }}-t_{1}^{\text {Ou }}=6915348 \mathrm{~s}$. ${ }^{\mathrm{f}}$ Perihelion on 9 September 2017 12:10:45.0 UTC (JD 2458006.007466) defines time $t_{0}=0 \mathrm{~s}$ for No. 4 ("Pseudo-MPEC" for 1I/“Oumuamua). Heliocentric distance $r_{1}^{\text {adj }}=r_{1}$ equated by adjusting $\chi_{\text {adj }}\{0\}$, cf. Equation (21)b. At $r_{1}$ it is assumed that $\dot{r}_{1}^{\text {adj }}=\dot{r}_{1}$, cf. Constraint (1). ${ }^{g}$ Anomalous accelerations relevant for orbit No. 4 with $\rho_{\odot}=4.9 \mathrm{~km}$. ${ }^{\mathrm{h}}$ The unmodified quantities relevant for orbit No. 4 are not repeated here. 


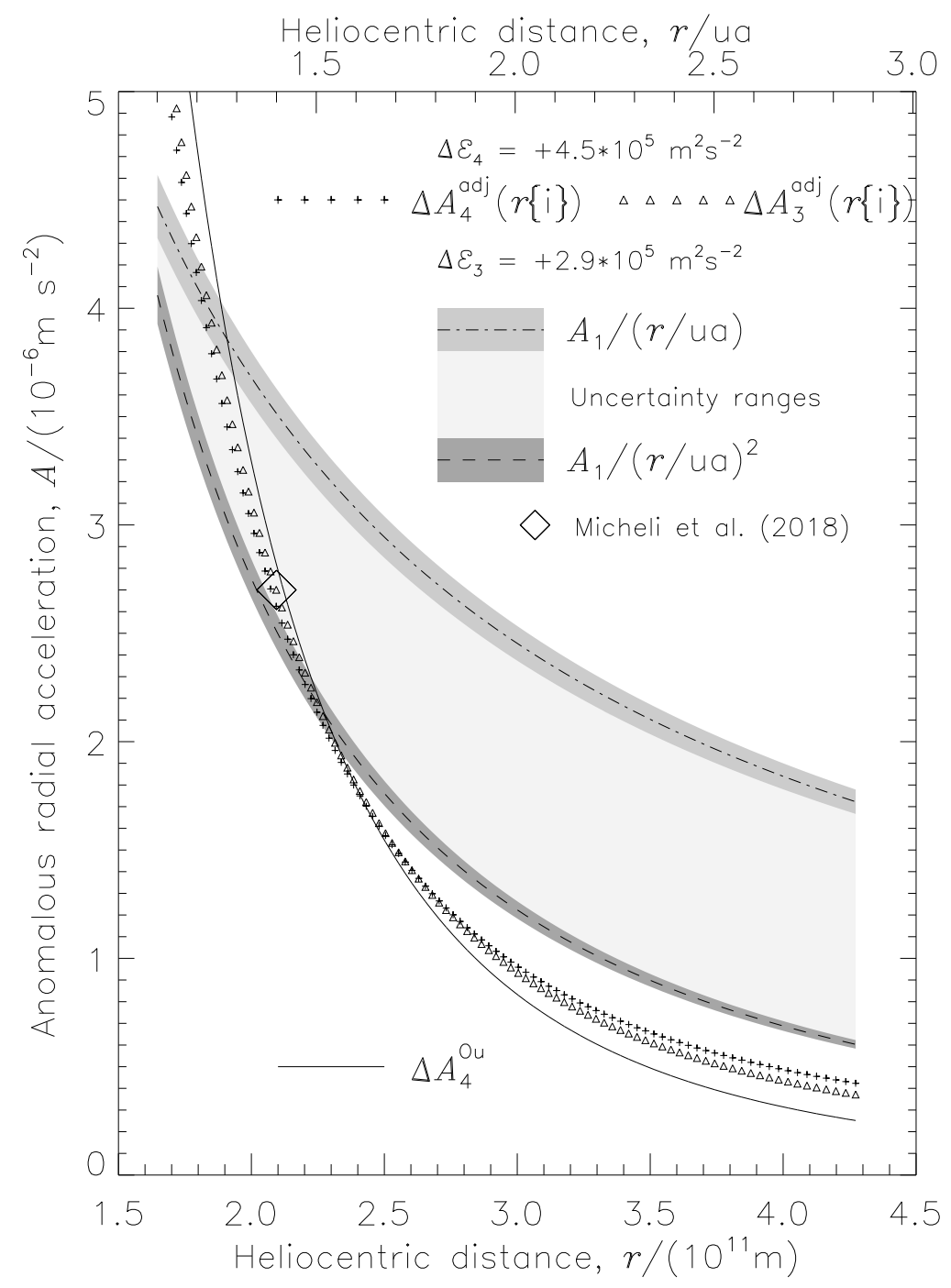

Figure 1. Shaded areas show the uncertainty ranges of the radial "non-gravitational acceleration" terms $A_{1} /(r / \mathrm{au})$ (dashed-dotted line) and $A_{1} /(r / \mathrm{au})^{2}$ (dashed line), cf. [8]. The residual radial accelerations $\Delta A_{3}^{\text {adj }}(r\{i\})$ and $\Delta A_{4}^{\text {adj }}(r\{i\})$ as differences between orbit solutions No. 3 and 4 and the corresponding adjusted trajectories are plotted as triangles and plus symbols, respectively, cf. Equation (26). Increases $\Delta \mathcal{E}_{3}$ and $\Delta \mathcal{E}_{4}$ of the specific energies provided the best overlap of the adjusted graphs with the $A_{1}$-terms and their uncertainty ranges. The diamond symbol indicates the anomalous radial acceleration $2.7 \times 10^{-6} \mathrm{~m} \mathrm{~s}^{-2}$ at $r_{\diamond}=1.4$ au (ref. [8]). An offset $\rho_{\odot}=100000 \mathrm{~km}$ would result in a residual radial acceleration $\Delta A_{4}^{\text {Ou }}$ shown as a solid line.

The task at hand now is to find specific energy increments $\Delta \mathcal{E}_{3}$ and $\Delta \mathcal{E}_{4}$ or offset values $\rho \odot$ Ou that lead to additional radial accelerations relative to orbits No. 3 and 4 in reasonable agreement with the range of "non-gravitational acceleration" found by Micheli et al. in ref. [8]. The results with different assumptions for $\Delta \mathcal{E}$ have been compared by many iterations with this range until acceptable fits with $\Delta \mathcal{E}_{3}=+2.9 \times 10^{5} \mathrm{~m}^{2} \mathrm{~s}^{-2}$ (for No. 3) and $\Delta \mathcal{E}_{4}=+4.5 \times 10^{5} \mathrm{~m}^{2} \mathrm{~s}^{-2}$ (for No. 4) were achieved as shown by the functions $\Delta A_{3,4}^{\text {adj }}$. The relative increase required in Equation (15) thus is $\approx 0.1 \%$.

\section{Discussion}

Since the expectation outlined in the introduction that an offset of the gravitational centre of the order of several kilometers would provide a direct explanation of 'Oumuamua's anomalous radial acceleration could not be substantiated, we pursued an indirect approach and proposed that the 
deviations from a Kepler orbit permitted us to assume wider uncertainty margins than listed in Table 1 for the orbit solutions No. 3 and 4 until reasonable fits could be produced. As shown in Figure 1, it was possible to obtain appropriate additional acceleration values covering the uncertainty range of ref. [8], but with a steeper decrease as a function of radial distance.

The offset values required to achieve such a fit with constant $\mathcal{E}_{\infty}$ and perihelion $q$ are far outside the range in line with the anomalous perihelion data mentioned in the introduction. The corresponding calculations were, nevertheless, helpful in showing that-even for large offset values-the deviations of the modified orbits from Kepler orbits on the data arcs is so small that the approximation in Equation (20) is very good. In Equation (12), for instance, the correction factors for the potential energy term would be $r_{1} /\left(r_{1}-\rho_{\odot}\right)=0.99999997$ and $\left(r_{1}-\rho_{\odot}^{\mathrm{Ou}}\right) / r_{1}=0.99939282$ at the start of the observations.

The orbits No. 1 and 4 together with the adjusted trajectories during the observation times have been plotted in Figure 2 with the help of Equations (21)c and (21)d. In addition, the trajectories have been traced back to their perihelia by decreasing $\chi$ in equidistant steps to zero. It can be seen that orbit No. 4 can barely be distinguished from the adjusted path on this scale. The main difference is indeed the additional acceleration and, consequently, a higher radial velocity at the end of the data arc combined with a greater radial distance. We find from Table 3 for orbit solution No. 3 a velocity $\dot{r}_{2}^{\text {adj }}$ higher by $5.9 \mathrm{~m} \mathrm{~s}^{-1}$ relative to $\dot{r}_{2}$ and $10.1 \mathrm{~m} \mathrm{~s}^{-1}$ for solution No. 4. This corresponds to an increase in the radial distance of $r_{2}^{\text {adj }}-r_{2}=49043 \mathrm{~km}$ for solution No. 4 and $21270 \mathrm{~km}$ for No. 3. The reason for the different results is that alternate initial conditions have been adopted for the adjustments No. 3 and 4. A boost of $40000 \mathrm{~km}$ has been deduced at the end of the observations on 2 January 2018 with NASA's Hubble Space Telescope (cf. https:/ / www.jpl.nasa.gov/ news / news.php?feature=7173, last accessed on 11 February 2019).

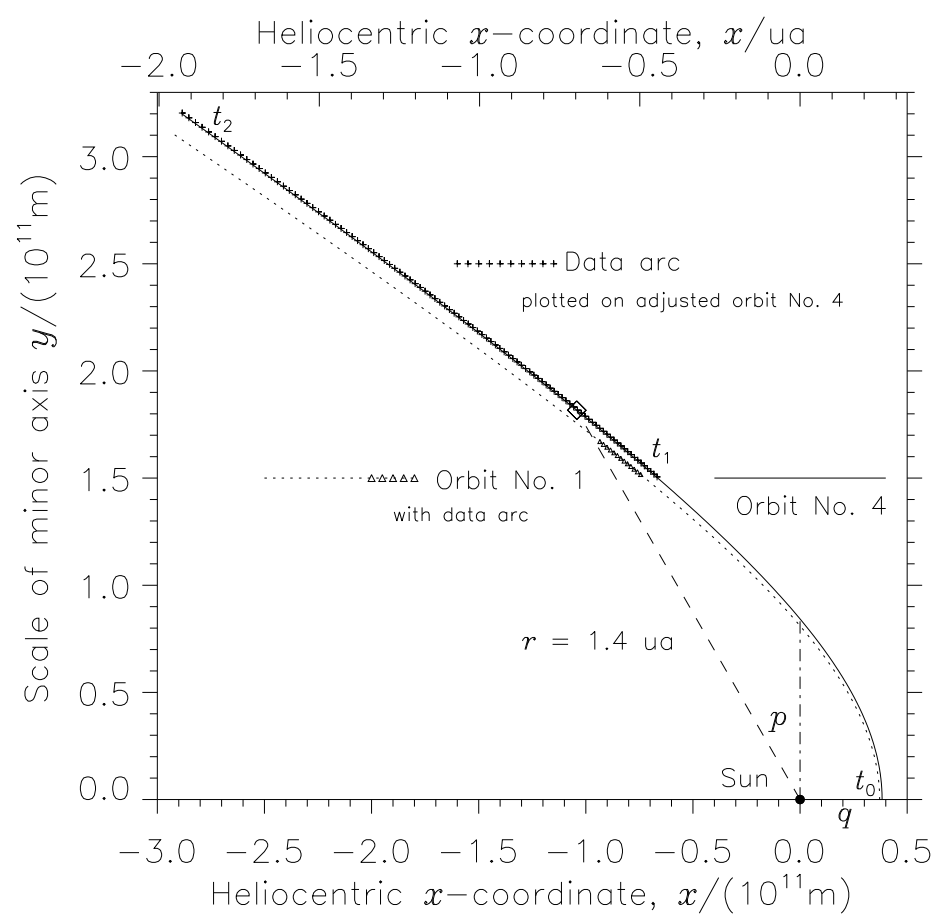

Figure 2. Hyperbolic orbit solutions for 'Oumuamua (post perihelion) (cf. Tables 1-3 ). Perihelion distance $q$; semi-latus rectum $p$. orbit No. 1 : Observations from 18 to 24 October 2017. The time interval with observations is indicated near $t_{1}$. Orbit No. 4: Observations from 14 October 2017, 10:32:41 UTC $\left(t_{1}\right)$ to 2 January 2018, 11:28:29 UTC $\left(t_{2}\right)$. Perihelion on 9 September 2017, 12:10:45 UTC $\left(t_{0}\right)$ (Minor Planet Center). The data arc is shown on the adjusted trajectory from time $t_{1}$ to $t_{2}$. The heliocentric distance $r_{\diamond}=1.4$ au is indicated. 
The adjusted specific energy $\mathcal{E}_{\text {adj }}$ and the angular momentum $\mathcal{M}_{\text {adj }}$ in Table 2 correspond to an increase in $V_{\infty}$ of $10.98 \mathrm{~m} \mathrm{~s}^{-1}$ for No. 3 and $17.04 \mathrm{~m} \mathrm{~s}^{-1}$ for No. 4 (relative variations $0.0416 \%$ and $0.0645 \%$, respectively). The impact parameters $h$ experienced relative increases of $\approx 0.0721 \%$ (No. 3) and $\approx 0.0381 \%$ (No. 4). The adjusted energy and angular momentum quantities are greater than the maximal values $\mathcal{E}_{\max }$ and $\mathcal{M}_{\max }$ allowed within the $1 \sigma$-limits listed in Table 1 . However, this should not necessarily be seen as a conflict, because of the modified solar gravitational potential in Equation (4). As a consequence of the offset $\rho_{\odot}$, the trajectory of 'Oumuamua is not an exact Kepler orbit and wider uncertainty ranges can be expected. The arguments presented in Section 2.2 also support this conjecture.

\section{Conclusions}

The observed anomalous acceleration has been modelled with respect to the orbit solutions JPL 16 and "Pseudo-MPEC" for $1 \mathrm{I} /$ 'Oumuamua. with the assumption that the trajectory of 'Oumuamua is not an exact Kepler orbit allowing us to assume angular momentum and approach energy values $\mathcal{M}_{\text {adj }}$ and $\mathcal{E}_{\text {adj }}$ outside the published uncertainty ranges.

In conclusion, it appears that the observed anomalous acceleration of the interstellar asteroid 1I/2017 U1 ('Oumuamua) can be modelled without the assumption of any cometary activity.

Author Contributions: Both the authors participated equally in developing the physical concept and formulation of the problem. Computation was done at the Max Planck Institute for Solar System Research in Göttingen by K.W. Both authors have read and agreed to the published version of the manuscript.

Funding: This research received no external funding.

Acknowledgments: We thank the Max-Planck-Institut für Sonnensystemforschung, Göttingen, and the Indian Institute of Technology (Banaras Hindu University) for organizational support and Peter Czechowsky for constructive criticism of the manuscript. This research has made extensive use of the Astrophysics Data System (ADS), as well as of data provided by the International Astronomical Union's Minor Planet Center and the JPL Small-Body Database. We thank the anonymous referees for their constructive comments and suggestions which improved the manuscript.

Conflicts of Interest: The authors declare no conflict of interest.

\section{References}

1. Meech, K.J.; Weryk, R.; Micheli, M.; Kleyna, J.T.; Hainaut, O.R.; Jedicke, R.; Wainscoat, R.J.; Chambers, K.C.; Keane, J.V.; Petric, A.; et al. A brief visit from a red and extremely elongated interstellar asteroid. Nature 2017, 552, 378-381. [CrossRef] [PubMed]

2. Knight, M.M.; Protopapa, S.; Kelley, M.S.P.; Farnham, T.L.; Bauer, J.M.; Bodewits, D.; Feaga, L.M.; Sunshine, J.M. On the rotation period and shape of the hyperbolic asteroid 1I/‘Oumuamua (2017 U1) from its lightcurve. Astrophys. J. 2017, 851, L31. [CrossRef]

3. Belton, M.J.S.; Hainaut, O.R.; Meech, K.J.; Mueller, B.E.A.; Kleyna, J.T.; Weaver, H.A.; Buie, M.W.; Drahus, M.; Guzik, P.; Wainscoat, R.J. The excited spin state of 1I/2017 U1 ‘Oumuamua. Astrophys. J. 2018, 856, L21. [CrossRef]

4. Katz, J.I. Why is interstellar object 1I/2017 U1 ('Oumuamua) rocky, tumbling and possibly very prolate? Mon. Not. R. Astron. Soc. 2018, 478, L95-L98. [CrossRef]

5. Drahus, M.; Guzik, P.; Waniak, W.; Handzlik, B.; Kurowski, S.; Xu, S. Tumbling motion of 1I/'Oumuamua and its implications for the body's distant past. Nat. Astron. 2018, 2, 407-412. [CrossRef]

6. Zhou, W.H. 'Oumuamua's rotation with the mechanical torque produced by interstellar medium. Astrophys. J. 2020, 899, 42. [CrossRef]

7. Kwiecinski, J.A.; Krause, A.L.; Van Gorder, R.A. Effects of tidal torques on 1I/2017 U1 (‘Oumuamua). Icarus 2018, 311, 170-174. [CrossRef]

8. Micheli, M.; Farnocchia, D.; Meech, K.J.; Buie, M.W.; Hainaut, O.R.; Prialnik, D.; Schörghofer, N.; Weaver, H.A.; Chodas, P.W.; Kleyna, J.T.; et al. Non-gravitational acceleration in the trajectory of 1I/2017 U1 ('Oumuamua). Nature 2018, 559, 223-226. [CrossRef] 
9. Bannister, M.T.; Bhandare, A.; Dybczyński, P.A.; Fitzsimmons, A.; Guilbert-Lepoutre, A.; Jedicke, R.; Knight, M.M.; Meech, K.J.; McNeill, A.; Pfalzner, S.; et al. The natural history of 'Oumuamua. Nat. Astron. 2019, 3, 594-602.

10. Trilling, D.E.; Mommert, M.; Hora, J.L.; Farnocchia, D.; Chodas, P.; Giorgini, J.; Smith, H.A.; Carey, S.; Lisse, C.M.; Werner, M.; et al. Spitzer observations of interstellar object 1I/‘Oumuamua. Astrophys. J. 2018, 156, 261. [CrossRef]

11. Katz, J.I. Evidence against non-gravitational acceleration of 1I/2017 U1 ‘Oumuamua. Astrophys. Space Sci. 2019, 364, 51 [CrossRef]

12. Ye, Q.-Z.; Zhang, Q.; Kelley, M.S.P.; Brown, P.G. 1I/2017 U1 ('Oumuamua) is hot: Imaging, spectroscopy, and search of meteor activity. Astrophys. J. 2017, 851, L5. [CrossRef]

13. Fitzsimmons, A.; Snodgrass, C.; Rozitis, B.; Yang, B.; Hyland, M.; Seccull, T.; Bannister, M.T.; Fraser, W.C.; Jedicke, R.; Lacerda, P. Spectroscopy and thermal modelling of the first interstellar object 1I/2017 U1 'Oumuamua. Nat. Astron. 2018, 2, 133-137. [CrossRef]

14. Meech, K.J. Ice can survive an interstellar trip. Nat. Astron. 2018, 2, 112-113. [CrossRef]

15. Hainaut, O.R.; Meech, K.J.; Micheli, M.; Belton, M.S.J. Rendezvous with 'Oumuamua. Messenger 2018, 173, 13-16.

16. Jewitt, D.C. From cradle to grave: The rise and demise of the comets. In Comets II, Part VII; Festou, M., Keller, H.U., Weaver, H.A., Eds.; University of Arizona Press: Tucson, AZ, USA, 2005; pp. 659-676.

17. Blum, J.; Schräpler, R.; Davidsson, B.J.R.; Trigo-Rodríguez, J.M. The physics of protoplanetesimal dust agglomerates. I. Mechanical properties and relations to primitive bodies in the Solar System. Astrophys. J. 2006, 652, 1768-1781. [CrossRef]

18. Beitz, E.; Blum, J.; Parisi, M.G.; Trigo-Rodríguez, J. The collisional evolution of undifferentiated asteroids and the formation of chondritic meteoroids. Astrophys. J. 2016, 824, 12. [CrossRef]

19. Trigo-Rodríguez, J.M.; Blum, J. Tensile strength as an indicator of the degree of primitiveness of undifferentiated bodies. Planet. Space Sci. 2009, 57, 243-249. [CrossRef]

20. Rafikov, R.R. Spin evolution and cometary interpretation of the interstellar minor object 1I/2017 ‘Oumuamua. Astrophys. J. 2018, 867, L17. [CrossRef]

21. Zuluaga, J.I.; Sánchez-Hernández, O.; Sucerquia, M.; Ferrín, I. A general method for assessing the origin of interstellar small bodies: The case of 1I/2017 U1 ('Oumuamua). Astron. J. 2018, 155, 236. [CrossRef]

22. Sekanina, Z. Outgassing as trigger of $1 \mathrm{I} /{ }^{\prime} O$ Oumuamua's nongravitational acceleration: Could this hypothesis work at all? arXiv 2019, arXiv:1905.00935.

23. Moro-Martín, A. Could 1I/‘Oumuamua be an icy fractal aggregate? Astrophys. J. 2019, 872, L32. [CrossRef]

24. Seligman, D.; Laughlin, G.; Batygin, K. On the anomalous acceleration of 1I/2017 U1 'Oumuamua. Astrophys. J. 2019, 876, L26. [CrossRef]

25. Mashchenko, S. Modelling the light curve of 'Oumuamua: Evidence for torque and disc-like shape. Mon. Not. R. Astron. Soc. 2019, 489, 3003-3021. [CrossRef]

26. Zhang, Y.; Lin, D.N.C. Tidal fragmentation as the origin of 1I/2017 U1 ('Oumuamua). Nat. Astron. 2020, 4, 852-860. [CrossRef]

27. Hui, M.-T.; Knight, M.M. New insights into interstellar object 1I/2017 U1 ('Oumuamua) from SOHO/STEREO nondetections. Astrophys. J. 2020, 896, L8. [CrossRef]

28. Seligman, D.; Laughlin, G. Evidence that 1I/2017 U1 ('Oumuamua) was composed of molecular hydrogen ice. Astrophys. J. 2020, 896, L8. [CrossRef]

29. Hoang, T.; Loeb, A. Destruction of molecular hydrogen ice and implications for 1I/2017 U1 ('Oumuamua). Astrophys. J. 2020, 899, L23. [CrossRef]

30. Flekkøy, E.G.; Luu, J.; Toussaint, R. The interstellar object 'Oumuamua as a fractal dust aggregate. Astrophys. J. 2019, 885, L41. [CrossRef]

31. Luu, J.X.; Flekkøy, E.G.; Toussaint, R. 'Oumuamua as a cometary fractal aggregate: The “Dust Bunny" model. Astrophys. J. 2020, 900, L22. [CrossRef]

32. Vazan, A.; Sari, R. On the aspect ratio of 'Oumuamua: Less elongated shape for irregular surface properties. Mon. Not. R. Astron. Soc. 2020, 493, 1546-1552. [CrossRef]

33. Bialy, S.; Loeb, A. Could solar radiation pressure explain 'Oumuamua's peculiar acceleration? Astrophys. J. 2018, 868, L1. [CrossRef] 
34. McNeill, A.; Trilling, D.E.; Mommert, M. Constraints on the density and internal strength of 1I/‘Oumuamua. Astrophys. J. 2018, 857, L1. [CrossRef]

35. Carry, B. Density of asteroids. Planet. Space Sci. 2012, 73, 98-118. [CrossRef]

36. Harp, G.R.; Richards, J.; Jenniskens, P.; Shostak, S.; Tarter, J.C. Radio SETI observations of the interstellar object 'Oumuamua. Acta Astronaut. 2019, 155, 51-54. [CrossRef]

37. Bailer-Jones, C.A.L.; Farnocchia, D.; Meech, K.J.; Brasser, R.; Micheli, M.; Chakrabarti, S.; Buie, M.W.; Hainaut, O.R. Plausible home stars of the Interstellar Object 'Oumuamua found in Gaia DR2. Astrophys. J. 2018, 156, 205. [CrossRef]

38. Hallatt, T.; Wiegert, P. The dynamics of interstellar asteroids and comets within the galaxy: An assessment of local candidate source regions for 1I/‘Oumuamua and 2I/Borisov. Astron. J. 2020, 159, 147. [CrossRef]

39. Higuchi, A.; Kokubo, E. Hyperbolic orbits in the Solar System: Interstellar origin or perturbed Oort cloud comets? Mon. Not. R. Astron. Soc. 2020, 492, 268-275. [CrossRef]

40. Lämmerzahl, C.; Preuss, O.; Dittus, H. Is the physics within the Solar System really understood? In Lasers, Clocks and Drag-Free Control: Exploration of Relativistic Gravity in Space; Dittus, H., Lämmerzahl, C., Turyshev, S.G., Eds.; Springer: Berlin, Germany, 2008; p. 75.

41. Anderson, J.D.; Campbell, J.K.; Ekelund, J.E.; Ellis, J.; Jordan, J.F. Anomalous orbital-energy changes observed during spacecraft flybys of Earth. Phys. Rev. Lett. 2008, 100, 091102-1-091102-4. [CrossRef]

42. Nieto, M.M.; Anderson, J.D. Earth flyby anomalies. Phys. Today 2009, 62, 76-77. [CrossRef]

43. Anderson, J.D.; Nieto, M.M. Astrometric solar-system anomalies. Relativity in Fundamental Astronomy: Dynamics, Reference Frames, and Data Analysis. Proc. IAU Symp. 2010, 261, 189-197.

44. Iorio, L. Gravitational anomalies in the Solar System? Int. J. Mod. Phys. D 2015, 24, 1530015. [CrossRef]

45. Cahill, R.T. Resolving spacecraft Earth-flyby anomalies with measured light speed anisotropy. Prog. Phys. 2008, 4, 9-15.

46. McCulloch, M.E. Modelling the flyby anomalies using a modification of inertia. Mon. Not. R. Astron. Soc. 2008, 389, L57-L60. [CrossRef]

47. Hasse, W.; Birsin, E.; Haehnel, P. On force-field models of the spacecraft flyby anomaly. arXiv 2009, arXiv:0903.0109.

48. Iorio, L. The effect of General Relativity on hyperbolic orbits and its application to the flyby anomaly. Schol. Res. Exch. 2009, 2009, 807695. [CrossRef]

49. Atchison, J.A.; Peck, M.A. Lorentz accelerations in the Earth flyby anomaly. J. Guid. Contr. Dynam. 2010, 33, 1115-1122. [CrossRef]

50. Wilhelm, K.; Wilhelm, H.; Dwivedi, B.N. An impact model of Newton's law of gravitation. Astrophys. Space Sci. 2013, 343, 135-144. [CrossRef]

51. Wilhelm, K.; Dwivedi, B.N. Impact models of gravitational and electrostatic forces. In Planetology; Palaszewski, B., Ed.; IntechOpen: London, UK, 2020.

52. Bopp, K. Fatio de Duillier: De la cause de la pesanteur. Schr. Straßburger Wiss. Ges. Heidelb. 1929, 10, 19-66.

53. Gagnebin, B. De la cause de la pesanteur, Mémoire de Nicolas Fatio de Duillier. Not. Rec. R. Soc. Lond. 1949, 6, 105-124.

54. Zehe, H. Die Gravitationstheorie des Nicolas Fatio de Duillier. Arch. Hist. Exact Sci. 1983, 28, 1-23. [CrossRef]

55. Wilhelm, K.; Dwivedi, B.N. Anomalous Earth flybys of spacecraft. Astrophys. Space Sci. 2015, 358, 18. [CrossRef]

56. Wilhelm, K.; Dwivedi, B.N. A physical process of the radial acceleration of disc galaxies. Mon. Not. R. Astron. Soc. 2018, 474, 4723-4729. [CrossRef]

57. Wilhelm, K.; Dwivedi, B.N. On the radial acceleration of disc galaxies. Mon. Not. R. Astron. Soc. 2020, 494, 4015-4025. [CrossRef]

58. Wilhelm, K.; Dwivedi, B.N. Secular perihelion advances of the inner planets and asteroid Icarus. N. Astron. 2014, 31, 51-55. [CrossRef]

59. Prša, A.; Harmanec, P.; Torres, G.; Mamajek, E.E.; Asplund, M.; Capitaine, N.; Christensen-Dalsgaard, J.; Depagne, É.; Haberreiter, M.; Hekker, S.; et al. Nominal values for selected solar and planetary quantities: IAU 2015 Resolution B3. Astron. J. 2016, 152, 41. [CrossRef]

60. Landau, L.D.; Lifshitz, E.M. Mechanics (Third Edition), Course of Theoretical Physics; Pergamon Press: Oxford, UK; New York, NY, USA; Toronto, ON, Canada; Sydney, Australia; Paris, France; Frankfurt, Germany, 1976; Volume 1. 
61. Bureau International des Poids et Mesures (BIPM). Le Système International d'Unités (SI), 9th ed.; BIPM: Sévres, France, 2019.

Publisher's Note: MDPI stays neutral with regard to jurisdictional claims in published maps and institutional affiliations.

(C) 2020 by the authors. Licensee MDPI, Basel, Switzerland. This article is an open access article distributed under the terms and conditions of the Creative Commons Attribution (CC BY) license (http://creativecommons.org/licenses/by/4.0/). 